\title{
SISTEMA DE POSICIONAMIENTO DE UNA ANTORCHA UTILIZADA EN LA APLICACIÓN DE RECUBRIMIENTOS POR PROYECCIÓN TÉRMICA
}

\section{(Positioning system of a torch used in thermal spray coatings applications)}

\author{
Edgar Absalón Torres Barahona', Carlos Mauricio Moreno Téllez², María Luisa Pinto Salamanca, \\ Leydi Julieta Cárdenas ${ }^{4}$, Juan David Fuentes Mojica ${ }^{5}$ \\ 1, 3 Programa de Ingeniería Electromecánica, Universidad Pedagógica y Tecnológica de Colombia, (Duitama- \\ Boyacá, Colombia). Grupo de Investigación en Energías y Nuevas Tecnologías GENTE. edgar.torres@uptc.edu. \\ co, marialuisa.pinto@uptc.edu.co \\ 2 Programa de Ingeniería Metalúrgica, Universidad Pedagógica y Tecnológica de Colombia. Colombia, \\ (Duitama-Boyacá, Colombia). carlosmauricio.moreno@uptc.edu.co \\ 4,5 Programa de Ingeniería Electromecánica, Universidad Pedagógica y Tecnológica de Colombia, Universidad \\ Pedagógica y Tecnológica de Colombia, (Duitama-Boyacá, Colombia). Grupo de Investigación en Energías y \\ Nuevas Tecnologías GENTE.
}

(Recibido mayo 16 de 2016 y aceptado junio 13 de 2016)

\begin{abstract}
Resumen
Se presenta el diseño, construcción y evaluación del desempeño de un sistema de posicionamiento utilizado para la deposición de recubrimientos con partículas fundidas o memifundidas, mediante el empleo de una antorcha CastoDyn Ds 8000 de proyección térmica con combustión de oxiacetileno. El diseño se ha hecho con parámetros obtenidos en el laboratorio de materiales de la Universidad Pedagógica y Tecnológica de Colombia, y la información determinada a partir de la evaluación del dispositivo, permite controlar las principales variables del proceso como la distancia de proyección, el flujo de polvo, la velocidad de la antorcha y la velocidad de rotación del porta muestras; esto se ha visto en los recubrimientos realizados en las pruebas de aplicación de circona/níquel sobre un sustrato de acero al carbono y analizados con Microscopia Electrónica de Barrido - MEB.
\end{abstract}

Palabras clave: Diseño Electromecánico, Recubrimiento por Proyección Térmica.

\begin{abstract}
This paper presents the design, construction and performance evaluation of a positioning system used for the deposition of coatings with molten particles, by using a torch CastoDyn Ds 8000 thermal spray with oxyacetylene combustion. The design has been done with parameters obtained in the laboratory of materials of the Universidad Pedagógica y Tecnológica de Colombia, and the information determined from the evaluation of the device, allows to control the main process variables as the projection distance, flow powder, torch speed and rotation speed of the sample holder; this has been seen in coatings made in application tests zirconia / nickel on a carbon steel substrate and analyzed with Scanning Electron Microscopy - SEM.
\end{abstract}

Key words: Thermal Spray Coating, Electromechanical Design.

Cómo citar este artículo:

Torres-Barahona, E.A., Moreno-Téllez, C.M., Pinto-Salamanca M.L., Cárdenas, L.J., \& Fuentes-Mojica, J.D. (2016). Sistema de posicionamiento de una antorcha utilizada en la aplicación de recubrimientos por proyección térmica. Revista Ingeniería Investigación y Desarrollo, 16 (2), pp. 55-65 


\section{INTRODUCCIÓN}

La corrosión, el desgaste y la fractura son considerados como algunos de los principales problemas en ingeniería, teniendo en cuenta que las fallas de los materiales acarrean pérdidas económicas y en múltiples circunstancias lesiones personales. Para prevenir estos efectos, se han desarrollado métodos de prevención dentro de los que se cuentan la aplicación de recubrimientos, o tratamientos superficiales a una estructura con el fin de que adquiera características específicas, diferentes a las del material base y que le permiten mejorar sus propiedades físico-químicas tales como la resistencia a la corrosión, al desgaste, la dureza, el aislamiento térmico o eléctrico entre otros.

Diferentes investigaciones en el mundo vienen aplicando nuevos materiales, así como perfeccionando técnicas y procedimientos para la aplicación de recubrimientos, con el fin de controlar y disminuir los problemas superficiales que implican desgaste y corrosión, ya que mejoran las características mecánicas, físicas y químicas, dando calidades superiores de funcionamiento a una superficie como se evidencia en los trabajos presentados en (González, 2008).

Una de las técnicas de aplicación de recubrimientos corresponde a la proyección térmica, definida como el proceso mediante el cual se aplica un recubrimiento metálico o no metálico a partir de la deposición de partículas fundidas o semifundidas, las cuales son aceleradas y proyectadas sobre la superficie de un material base o sustrato previamente preparado (Morales et al, 2012).

La técnica de proyección de polvos por llama, que utiliza gases de combustión como fuentes de calor y polvo como materia prima de recubrimiento, ofrece la ventaja de proyectar materiales no metálicos que no están disponibles en forma de varilla o alambre. Esta técnica es considerada como una potencial alternativa para la protección de dispositivos, dada su versatilidad y la disposición de un buen número de materiales a depositar sobre sustratos de diferente tamaño y forma, más si se tiene en cuenta que este proceso permite recubrir piezas de grandes dimensiones y diversas geometrías, siendo además un proceso económico, lo cual viabiliza su utilización en la aplicación de recubrimientos para diversos componentes industriales (Marulanda et al, 2014), (Restrepo et al, 2012), (Vargas, 2012).
El proceso de proyección térmica fue patentado en 1882 por M. U. Schoop (Zurich, Suiza), cuando trató de modificar un soplete de oxi-acetileno, antorchas posteriores fueron modificadas para aceptar materiales en polvo (Ružić, et al., 2012). Muchas de las mejoras en estos procesos se han hecho desde entonces, pero es después de la segunda guerra mundial, sobre todo en el período entre el 1960 y el 1990, cuando comenzó su amplia aplicación en diversas industrias (Davis, 2004). Hoy en día, existe un buen número de procesos para aplicar recubrimientos, así como un número casi ilimitado de materiales de recubrimiento; en aplicaciones industriales, generalmente la aplicación del recubrimiento térmico se hace a través de equipos automáticos.

Según un estudio de la Universidad Nacional, la industria colombiana aún no ha adoptado la proyección térmica como un método habitual para combatir la corrosión y el desgaste. En un noventa y cinco por ciento (95\%) la recuperación y el mantenimiento de piezas se hacen a través de procesos convencionales utilizando pinturas de alto desempeño y protección catódica. Además, la ausencia de estudios en esta área, genera ignorancia sobre los grandes beneficios de la proyección térmica (Marín, 2008).

Las investigaciones en esta técnica requieren un control riguroso de las variables de proyección, como son: la distancia de proyección, la velocidad de la antorcha, la velocidad de rotación del porta muestras, el flujo de los polvos y los gases de combustión, de tal forma que al gobernar las variables de proyección se pueda controlar las características del recubrimiento obtenido. Esto se logra mediante la implementación de sistemas automatizados, de control flexible, que se adapte a las características requeridas en la aplicación de los recubrimientos.

El Instituto para la Investigación y la Innovación en Ciencia y Tecnología de Materiales -INCITEMA de la Universidad Pedagógica y Tecnológica de Colombia, ha adquirido un equipo manual de proyección térmica, que incorpora tecnología de proyección térmica usando polvo, sin embargo para desarrollar investigaciones en la línea de recubrimientos se hace necesario controlar todos los parámetros que intervienen en el proceso lo cual se logra con el diseño e implementación de un dispositivo sistematizado. 
Este trabajo presenta los resultados de diseño, implementación y desempeño de un sistema de posicionamiento de una antorcha utilizada para la aplicación de recubrimiento por proyección térmica por combustión oxiacetilénica.

Como aporte principal se muestra un proceso de desarrollo de maquinaria y equipo desde la etapa de diseño hasta su validación. El trabajo corresponde con los resultados del proyecto: Optimización de un sistema de recubrimiento cerámico por spray térmico, código SGI 1526 UPTC del grupo de investigación GENTE.

\section{EL PROCESO DE RECUBRIMIENTO POR PROYEC- CIÓN TÉRMICA POR LLAMA}

En la proyección térmica por llama, los materiales metálicos o no metálicos finamente divididos, se depositan en un estado fundido o semifundido para formar un recubrimiento. El material de aporte puede estar en forma de polvo, alambre o materiales fundidos (García y Cuetos, 2001), (International Thermal Spray Association, 2015), el proceso de aplicación de recubrimiento se presenta en la Figura 1.

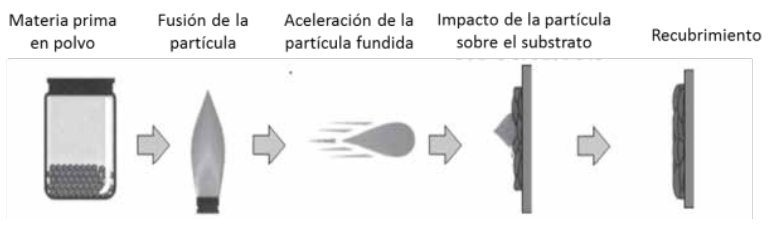

Figura 1. Etapas en la aplicación de un recubrimiento por proyección térmica (García y Cuetos., 2001)

El material de aporte en forma de polvo se funde en una llama de acetileno/oxígeno y con la ayuda de los gases de combustión en expansión es lanzado sobre la superficie de la pieza a tratar. El calor generado se debe la reacción química entre los gases de combustión, pudiendo alcanzar temperaturas hasta de $3100^{\circ} \mathrm{C}$ para generar la fusión de las partículas a depositar.

La capa de un recubrimiento se forma al proyectar pequeñas gotas del material fundido sobre un sustrato, las cuales tienen alta energía cinética y térmica, en el momento del impacto se deforman en forma de disco y se enfrían rápidamente formando un recubrimiento compacto por agregación sucesiva de partículas (ASM, 2003).

El anclaje o adherencia depende de la preparación superficial y ocurre por unión mecánica o metalúrgica entre el recubrimiento y el sustrato.

Las características de porosidad, dureza, rugosidad, resistencia al desgaste y a la corrosión de los recubrimientos obtenidos dependen del material depositado, el sistema de proyección térmica elegido y de los parámetros de proyección. (Morales et al, 2012).

\section{MATERIALES Y MÉTODOS}

\subsection{Materiales}

3.1.1 Software de diseño y funcionamiento de la máquina

El modelamiento mecánico de la máquina se hizo con base en el software Inventor de Autodesk versión educativa 2013. El diseño y desarrollo de las tarjetas de control se hizo con Proteus ${ }^{\mathrm{TM}}$ v.6; para la integración de componentes electrónicos y adquisición de datos se implementó un sistema de control e interfaz de usuario con LabView ${ }^{\mathrm{TM}}$ v.10 a través de la integración de librerías para el uso de la tarjeta Arduino MEGA como sistema físico para adquisición de datos y control.

\subsubsection{Polvos utilizados como material de aporte}

El desempeño del equipo se evaluó proyectando un recubrimiento bicapa, constituido por una capa de anclaje de una aleación base níquel y una capa superior de óxido de circonio sobre un sustrato de acero al carbono, al cual se le hizo una preparación previa con chorro abrasivo de corindón, obteniendo una rugosidad aproximada de $\mathrm{Ra}=5$ micras. Los recubrimientos fueron evaluados a partir de Microscopia Electrónica de Barrido -MEB, al igual que la composición elemental de los polvos y del sustrato. Ver Tabla 1. 
Tabla 1. Características de materiales de recubrimiento, obtenidas por SEM

\begin{tabular}{cccc}
\hline Material & Elementos & \% en Peso & \% Atómico \\
\hline \multirow{2}{*}{ Material base o } & Mn K & 0.32 & 0.53 \\
sustrato & Fe K & 60.31 & 99.47 \\
& Total & 60.62 & 100 \\
\hline \multirow{2}{*}{ Material de } & $\mathrm{O} \mathrm{K}$ & 14.87 & 72.91 \\
recubrimiento & $\mathrm{Al} \mathrm{K}$ & 4.86 & 14.13 \\
cerámico & Fe K & 0.85 & 1.19 \\
& Zr L & 13.70 & 11.78 \\
\hline Base de & Total & 34.28 & 100 \\
\hline \multirow{2}{*}{ adherencia } & Si K & 0.97 & 3.84 \\
& Fe K & 0.23 & 0.45 \\
& Ni K & 50.79 & 95.71 \\
& Total & 52.00 & 100 \\
\hline
\end{tabular}

Para la aplicación del recubrimiento se utilizó una antorcha de proyección térmica de referencia CastoDyn DS 8000, de la casa de Eutectic.Castolin, modificada y montada en El sistema electromecánico diseñado e implementado en este trabajo. La antorcha se muestra en la Figura 2.

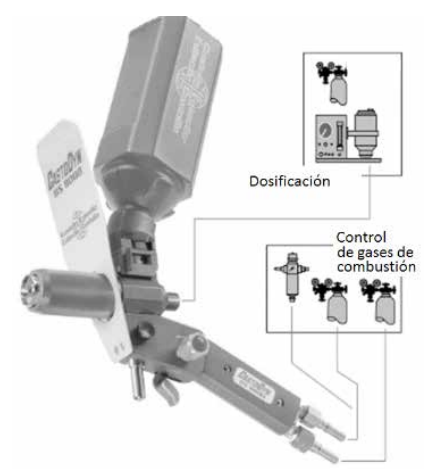

Figura 2. Antorcha utilizada para la proyección térmica por llama (Castolin Iberica s.a.)

\subsection{Métodos}

\subsubsection{Especificaciones generales de diseño}

El posicionamiento de la antorcha se realizó mediante el diseño y construcción de un equipo a partir de las especificaciones técnicas presentadas en la Tabla 2 definidas a partir de requerimientos del Instituto para la investigación e innovación en ciencia y tecnología de materiales -INCITEMA y criterios de diseño de los autores.

Tabla 2. Requerimientos del sistema

\begin{tabular}{|c|c|}
\hline CARACTERÍSTICA & ESPECIFICACIÓN \\
\hline Uso & $\begin{array}{l}\text { Aplicación de recubrimientos por } \\
\text { spray térmico }\end{array}$ \\
\hline Aplicación & Laboratorio de materiales \\
\hline Espacio de trabajo & $\begin{array}{l}\text { Aplicación de recubrimiento sobre } \\
\text { probetas de tamaño máximo de } \\
50 \mathrm{~cm} \text { de diámetro por } 50 \mathrm{~cm} \text { de } \\
\text { longitud. }\end{array}$ \\
\hline $\begin{array}{l}\text { Velocidades de } \\
\text { aplicación }\end{array}$ & $\begin{array}{l}\text { Velocidad relativa entre la boquilla } \\
\text { de proyección y el sustrato, variable } \\
\text { entre } 0 \text { y } 25 \mathrm{~m} / \mathrm{s}\end{array}$ \\
\hline Altura de proyección & Variable entre 0 y $20 \mathrm{~cm}$ \\
\hline $\begin{array}{l}\text { Desplazamiento } \\
\text { de la boquilla de } \\
\text { proyección }\end{array}$ & Horizontal en un espacio de $40 \mathrm{~cm}$. \\
\hline $\begin{array}{l}\text { Configuración } \\
\text { mecánica }\end{array}$ & $\begin{array}{l}\text { Uso de un sistema bidimensional para } \\
\text { el posicionamiento de la antorcha y } \\
\text { rotacional para el movimiento de la } \\
\text { probeta }\end{array}$ \\
\hline Dosificación de polvo & $\begin{array}{l}\text { Regulada y por arrastre de polvo } \\
\text { mediante la energía cinética de un } \\
\text { gas inerte. }\end{array}$ \\
\hline $\begin{array}{l}\text { Suministro de gases } \\
\text { de combustión }\end{array}$ & $\begin{array}{l}\text { Reguladas en flujo y con } \\
\text { accionamiento ON/OFF por medio de } \\
\text { electroválvulas }\end{array}$ \\
\hline $\begin{array}{l}\text { Temperatura de } \\
\text { proyección }\end{array}$ & $\begin{array}{l}\text { Con posibilidad de precalentamiento } \\
\text { del sustrato hasta } 350^{\circ} \mathrm{C}\end{array}$ \\
\hline
\end{tabular}

\subsubsection{Posicionamiento de la antorcha y del portamuestras}

El diseño del equipo tuvo en cuenta tres tipos de movimiento para el posicionamiento relativo entre la boquilla y el sustrato: i) uno vertical (eje y) que determina la distancia entre la boquilla de proyección y el sustrato, ii) un movimiento longitudinal (eje $\mathrm{x}$ ) que permite definir el rango de proyección y iii) un movimiento de giro para el porta muestras (rotación en el eje $\mathrm{x}$ ), que completa la aplicación. Los ejes de movimiento seleccionados se observan en la Figura 3 , con $h_{t}$ altura de trabajo, $v_{t}$ velocidad longitudinal y $w_{t}$ velocidad angular. 


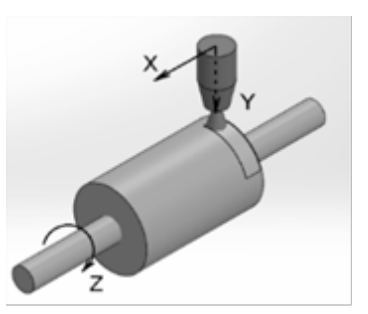

$a$.

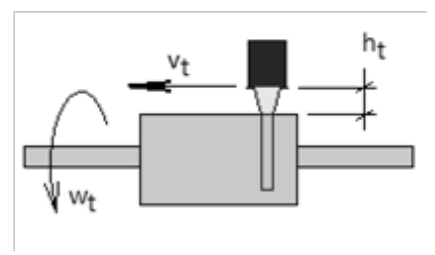

$b$.
Figura 3. a. referencial definido, b. movimientos y altura de trabajo

Se consideraron además las variables relacionadas con el control de temperatura del sustrato y de la llama y el flujo másico del material de aporte (polvos).

La llama se genera a partir de una mezcla de acetileno/ oxigeno la cual puede alcanzar temperaturas lo suficientemente altas capaces de fundir materiales de alto punto de fusión como los cerámicos. La relación de oxígeno - combustible puede variar desde una atmósfera carburante a una oxidante a partir de la aplicación de rotámetros como controladores de flujo volumétrico, se tiene en cuenta el uso de extintores de llama y procedimientos seguros tanto para el encendido como para la extinción de la misma, los cuales se hacen explícitos en un manual de operación.

El control del flujo de polvos se hizo mediante un distribuidor de polvos, el cual es presurizado con un gas inerte, cuyo flujo permite el arrastre y dosificación constante de polvo.

\subsubsection{Diseño de la máquina}

El sistema diseñado y construido para la aplicación del recubrimiento tiene en cuenta las especificaciones relacionadas en la Tabla 1 . El análisis de sus componentes se presenta a continuación:

Estructura: se diseñó con base en criterios de ergonomía para la manipulación de componentes y probetas, de estética y de funcionamiento; este último criterio tiene en cuenta la zona de trabajo, la ubicación de los dispositivos de control y la posición del dosificador. El resultado del modelo CAD de la estructura obtenido a partir de una etapa de diseño conceptual, se muestra en la Figura 4(a).
Movimiento Vertical: el desplazamiento sobre el eje vertical se realiza con velocidad constante partiendo de una posición de referencia y se ejecuta teniendo en cuenta el diámetro de la probeta y la altura de la boquilla sobre el sustrato. La relación de vueltas del motor y el desplazamiento está definida por:

$$
y=\frac{\theta}{2 \pi} p
$$

Siendo $y$ el desplazamiento vertical requerido, $\theta$ en ángulo de giro en radianes y $\mathrm{p}$ el paso del tornillo.

En la Figura 4(b) se visualiza el sistema vertical compuesto por un tornillo, dos ejes guía, y rodamientos lineales que permiten un movimiento suave y continuo. Además, este sistema hace posible un desplazamiento con la precisión y anclaje vertical en condición de reposo.

Movimiento horizontal: en la Figura 4(c) se esquematiza el dispositivo diseñado para el movimiento horizontal (eje $\mathrm{x}$ ), utilizando un tornillo sin fin, dos ejes guía, soportes y un carro de desplazamiento. El movimiento sobre este eje se realiza por medio de un motor paso a paso de $1,8^{\circ}$ por paso y un torque de 1.41 N.m, y su desplazamiento está definido por la relación:

$$
x=\frac{n}{N} p
$$

Siendo $x$ el desplazamiento, $N$ el número de pasos por vuelta del motor, $n$ en número de pasos programados del motor y $p$ el paso del tornillo.

La integración del sistema horizontal y vertical se muestra en la Figura 6(d); este ensamble da rigidez y estabilidad a cada uno de los sistemas, permitiendo la precisión requerida en los desplazamientos.

Movimiento de giro: en la Figura 4(e) se muestra el acople entre el motor y la copa que se emplea para fijar las probetas a recubrir. El motor utilizado cuenta con un variador de velocidad que permite múltiples niveles de rotación de acuerdo al tipo de recubrimiento, requiriendo velocidades que varían entre 50 rpm y 500 rpm.

Dosificación: el suministro de polvo de recubrimiento se hizo de manera regulada a través de un dispositivo

Rev. I2+D. Vol.16 N². Julio-Diciembre. 2016. Sogamoso-Boyacá, Colombia. 
que contiene un plato ranurado que toma el polvo de un depósito vertical y lo transporta a una salida, donde por fenómeno de arrastre a partir de la energía cinética de un gas inerte, se dosifica el polvo en la cantidad y tiempo requerido. En la Figura 5 se muestra el dosificador diseñado. Para el movimiento del plato ranurado se utilizó un motor DC de $24 \mathrm{~V}, 100 \mathrm{~W}$, que permite un movimiento de velocidad controlada para regular la dosificación. Para el control de flujo de aire, gas inerte y gases de combustión se utilizaron electroválvulas de tipo ON/OFF con presión de trabajo de $1 \mathrm{MPa}$ y la regulación de caudal del argón, oxígeno y acetileno se hizo mediante rotámetros de 50, 60 y 150 SCFH respectivamente; la presión de trabajo del acetileno y el oxígeno incluyó válvulas reguladoras de presión de 1.47 MPa y $6 \mathrm{~m} 3 / \mathrm{h}$.

a.

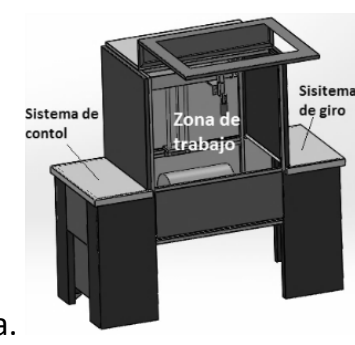

b.
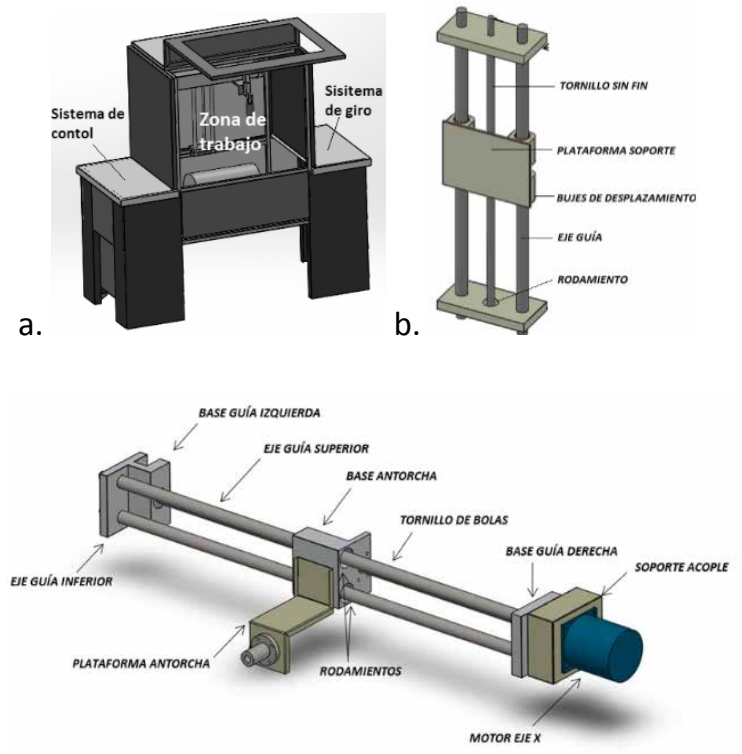

C.

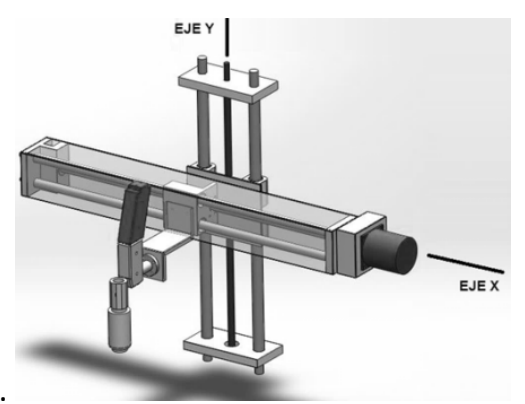

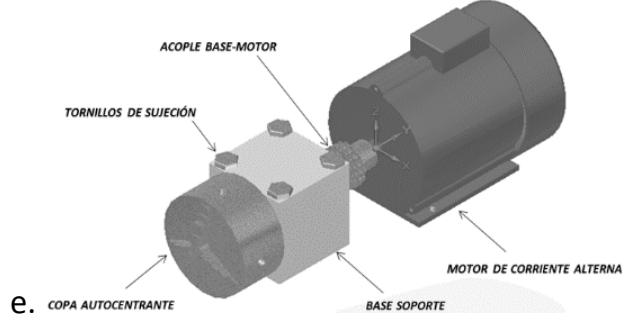

Figura 4. Modelos CAD de la máquina diseñada. a. Estructura. b. Mecanismo de movimiento vertical. c. Mecanismo de movimiento horizontal. d. Ensamble mecanismos horizontal y vertical. e. Mecanismo de movimiento de giro

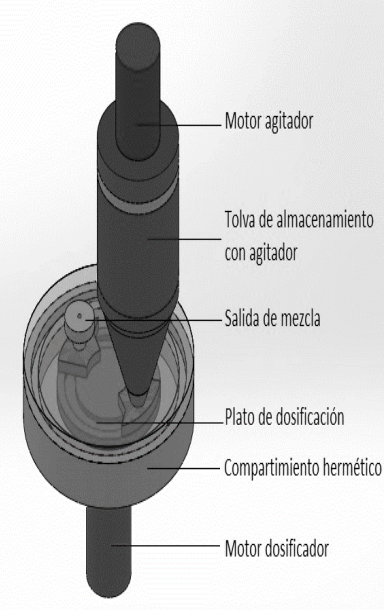

Figura 5. Dosificador de polvo de recubrimiento

\subsubsection{Pruebas desarrolladas}

La validación del diseño e implementación del sistema se hizo mediante tres pruebas de funcionamiento:

\section{Prueba 1: Funcionamiento General.}

En cuanto al funcionamiento mecánico, se diseñaron y desarrollaron pruebas de movimiento vertical, horizontal, de giro, de dosificación y de gobierno de las electroválvulas.

Movimiento vertical: gobernado por un moto-reductor DC, fue controlado por una acción ON/OFF y la posición se definió por la acción redundante entre un encoder incremental y el tiempo transcurrido; los límites de trabajo estaban limitados por sensores de fin de curso. 
Movimiento horizontal: gobernado por un motor paso a paso, que actúa sobre un tornillo sin fin, determinó su posición mediante la relación de avance por paso.

Movimiento de giro: dado por un motor trifásico de (559W, 220V), controla la velocidad por la acción de un variador gobernado por una entrada análoga de voltaje, cuya relación fue determinada por la relación velocidad angular versus voltaje de entrada.

El control del dosificador de polvo: se hizo mediante acciones de control de modulación de ancho de pulso PWM sobre los motores del agitador, las pruebas de dosificación relacionan el material dosificado en función de ciclo de trabajo de la señal modulada.

Finalmente, para controlar el flujo de gases se verificó la acción de un circuito de control de fase implementado con tiristores BTA24 de 600V, 25 A, con acoplamiento óptico, para regular la apertura y cierre de cuatro electroválvulas de 120V, 150psi.

\section{Prueba 2: Aplicación de recubrimiento.}

Para el análisis del sistema en la aplicación del recubrimiento se utilizó la relación de materiales previamente presentados en la Tabla 1 y se desarrollaron pruebas de ancho del cordón proyectado versus altura de la boquilla, espesor de recubrimiento versus altura de proyección y características del material depositado. Se tiene en cuenta que la temperatura máxima de la llama va desde $2700^{\circ} \mathrm{C}$ a $3100^{\circ} \mathrm{C}$ y la velocidad de movimiento lineal de la antorcha entre $80 \mathrm{~mm} / \mathrm{s}$ y 100 $\mathrm{mm} / \mathrm{s}$ (González, 2008).

\section{ANÁLISIS DE RESULTADOS}

\subsection{Máquina Construida}

Para la implementación del sistema se tuvo en cuenta el diseño y la construcción de los componentes de acuerdo con las especificaciones técnicas relacionadas con dimensiones, tolerancias y acabados. Una visión general del equipo se muestra en la Figura 6.

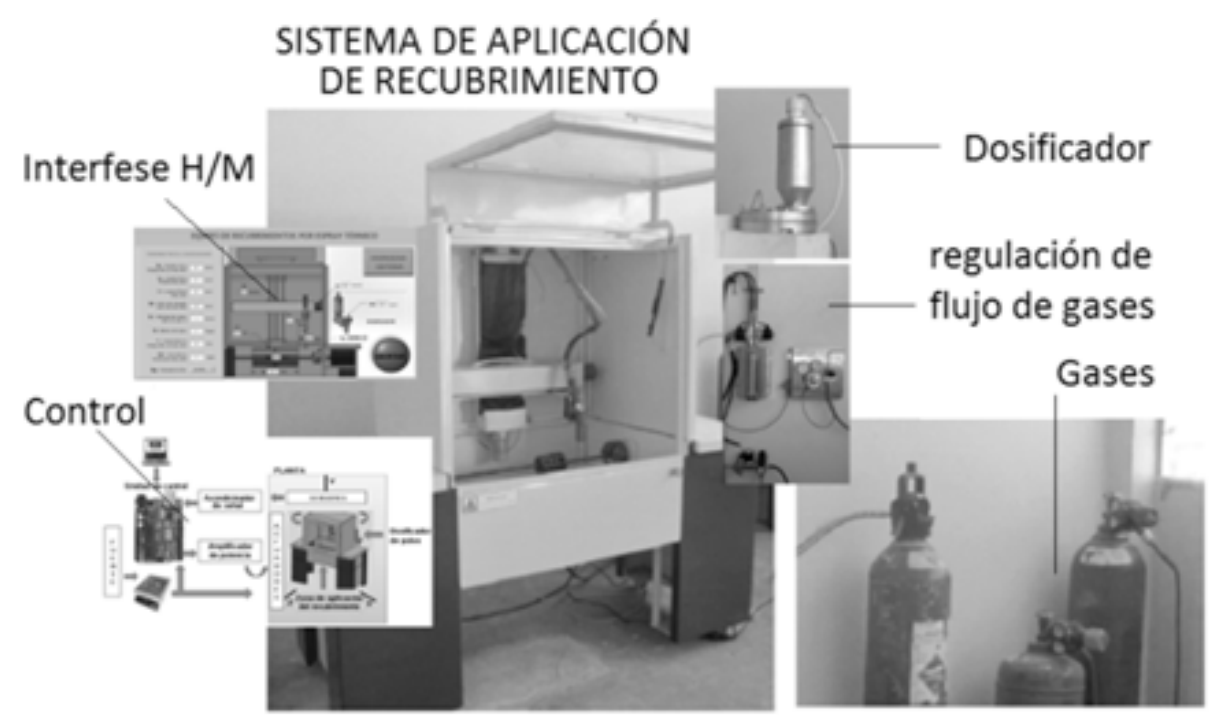

Figura 6. Sistema construido para la aplicación de recubrimiento por proyección térmica

\subsection{Desempeño del Sistema}

\subsubsection{Movimiento vertical}

En la Figura 7(a) se muestra el comportamiento lineal de posición versus tiempo, que valida la correspondencia entre la posición obtenida por tiempo transcurrido y la posición censada a través de un encoder incremental, cuya relación de posición con respecto al número de pulsos está determinado por la curva mostrada en la Figura 7b. Se comprobó que se cumple la altura de trabajo de la boquilla con respecto al sustrato, variable fundamental en la aplicación del recubrimiento.

Rev. I2+D. Vol.16 N². Julio-Diciembre. 2016. Sogamoso-Boyacá, Colombia. 


\subsubsection{Movimiento horizontal}

Las pruebas hechas al sistema de movimiento horizontal muestran un comportamiento lineal entre el número de pasos programado y la posición de la antorcha, en el rango de trabajo definido de $400 \mathrm{~mm}$. La velocidad máxima se obtuvo a una frecuencia de pulsos de control del motor de $800 \mathrm{~Hz}$, lográndose un rango de valores de velocidad entre 0 y $25 \mathrm{~mm} / \mathrm{s}$. En cuanto al cumplimiento de longitud de trabajo, se obtuvo un desplazamiento efectivo de $450 \mathrm{~mm}$ entre los sensores instalados del tipo fin de curso.

a.
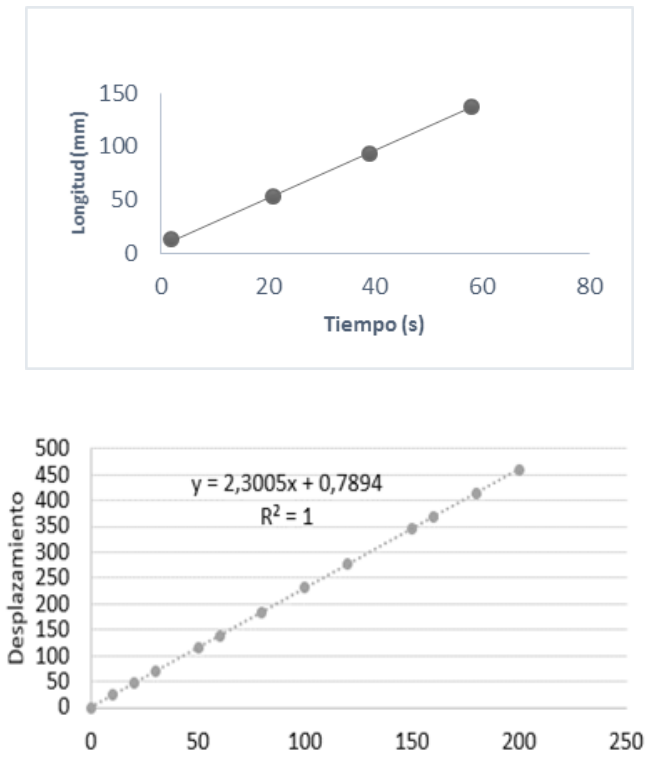

b.

Número de pulsos

Figura 7. Calibración movimiento vertical. a) Longitud de movimiento vs. Tiempo transcurrido, b) Longitud de movimiento vs. Pulsos del encoder

\subsubsection{Movimiento de giro}

Las pruebas realizadas al movimiento vertical mostraron un comportamiento lineal entre la velocidad programada y la velocidad medida de forma experimental, sin embargo, estos valores no fueron correspondientes en valor, por lo que se obtuvo una curva de calibración por regresión lineal (ver Figura 8), encontrándose la relación:

$$
v_{\text {medida }}=3,573 v_{\text {programada }}-57,338
$$

A partir de este análisis se realizó la reprogramación del sistema. Una vez hecha la corrección, se verifica el cumplimiento de la relación entre lo programado y la velocidad de salida, obteniéndose valores coincidentes.

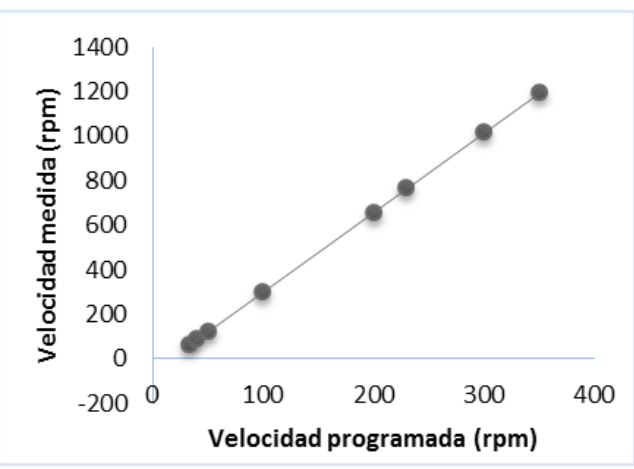

Figura 8. Curva de calibración movimiento de giro

\subsubsection{Dosificador}

Las pruebas hechas al dosificador de material de recubrimiento, relacionan la acción modulación por ancho de pulso PWM que controla el motor y que da movimiento angular del plato de dosificación, obteniendo la curva de calibración indicada en la Figura 9 , donde se puede observar un comportamiento lineal entre la velocidad angular del motor y el ciclo de trabajo de la señal aplicada. El control de este movimiento permite determinar el flujo másico de recubrimiento a partir de pruebas experimentales.

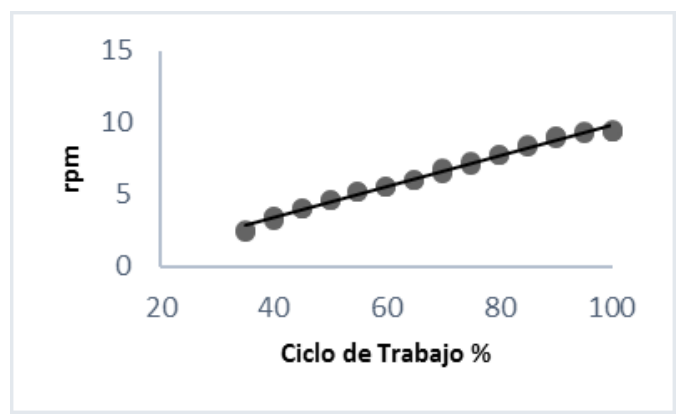

Figura 9. Curva de Calibración de Velocidad del plato de dosificación

\subsection{Aplicación del recubrimiento}

Al aplicar el recubrimiento se determina una relación entre altura de proyección $h_{t}$ y anchura del cordón puesto $p$ el cual determina la razón entre movimiento angular 
y lineal, Figura 10(a). Un análisis matemático determina que la aplicación de una capa de recubrimiento sin traslape se logra según la ecuación 4; la variación en la relación entre $\omega_{t}$ y $v_{t}$ permite definir el paso y con éste el traslape entre cordones adyacentes, como:

$$
\omega_{t}=\frac{2 \pi v_{t}}{p} \quad\left[\frac{R a d}{s}\right]
$$

Siendo $p$ el paso o ancho del cordón depositado, como se observa en la Figura 10(b).

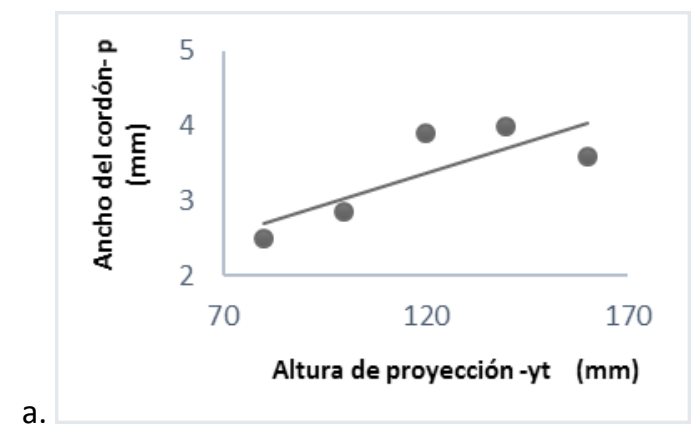

a.

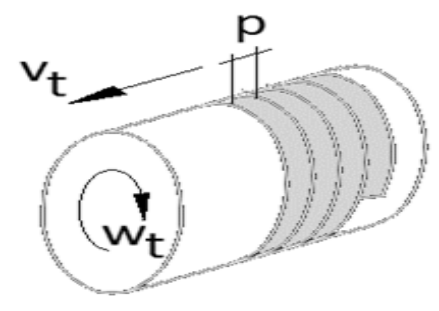

Figura 10. a. Relación altura vs ancho del cordón. b. Variables que intervienen

Con esta relación se establece la densidad superficial de aplicación de recubrimiento según el siguiente modelo matemático:

$$
\rho_{\mathrm{s}}=\frac{\mathrm{v}_{\mathrm{d}}}{\mathrm{v}_{\mathrm{t}} \sqrt{\mathrm{p}^{2}+\left(2 \pi \mathrm{r}_{\mathrm{p}}\right)^{2}}}
$$

Siendo $\mathrm{v}_{\mathrm{d}}$ el flujo másico de polvo, el cual está relacionado directamente con el flujo volumétrico por la densidad aparente. Si se tiene en cuenta que $m=\rho_{s} A$ y que igualmente $m=\rho_{v} V$, entonces se puede encontrar la densidad volumétrica y programar el espesor de forma preliminar de acuerdo con la relación:

$$
e=\frac{\rho_{s}}{\rho_{V}}[m m]
$$

Sin embargo, este es solo un método aproximado, tomado solo como referente para la definición de parámetros de operación del equipo, ya que el espesor es un factor que depende del aplanamiento de las partículas al impactar con el sustrato, de la porosidad que de esto se derive y las pérdidas de material por efecto de dispersión y rebote.

Un análisis del recubrimiento depositado con el equipo diseñado y hecho por Microscopia Electrónica de Barrido - SEM (ver Figura 11), muestra las características propias de un material depositado por proyección térmica. En la Figura 11(a) se observa el sustrato y la primera capa, con un espesor promedio de $66.22 \mu \mathrm{m}$ que sirve como anclaje.

En la Figura 11(b), se observa el espesor del recubrimiento, para el cual se hizo un análisis de espesor con respecto a la altura de proyección, manteniendo un flujo constante de polvo de $10.02 \mathrm{~g} / \mathrm{min}$, con un precalentamiento del sustrato de $250^{\circ} \mathrm{C}$.

Finalmente, en la Figura 11(c) se observan 4 puntos relacionados con las características propias del recubrimiento obtenido: 1- desprendimiento de material causados en el corte o pulido, 2- una partícula de polvo semifundida y rodeada por 3- material fundido.

\section{CONCLUSIONES}

De acuerdo con los resultados obtenidos, se dispone de un equipo flexible que puede ser utilizado para el desarrollo de recubrimientos por proyección térmica que responde a las especificaciones de diseño planteadas. Estos resultados permiten inferir la validez del sistema eléctrico, electrónico y de control implementados.

El posicionamiento automático y el control de flujo de gases permiten un protocolo y procedimiento seguros tanto para el encendido de llama como para la extinción de la misma; los procedimientos expresados en manuales relacionan el montaje y ubicación de la probeta, la alimentación del dosificado con el polvo de recubrimiento y el flujo másico de oxígeno, acetileno y del gas de arrastre de polvo. 
A partir del análisis de la información, diseño y desarrollo del equipo, se consolida una base del conocimiento que amplía los recursos del grupo de investigación, en lo referente a sistemas de control de movimiento y control numérico computarizado.
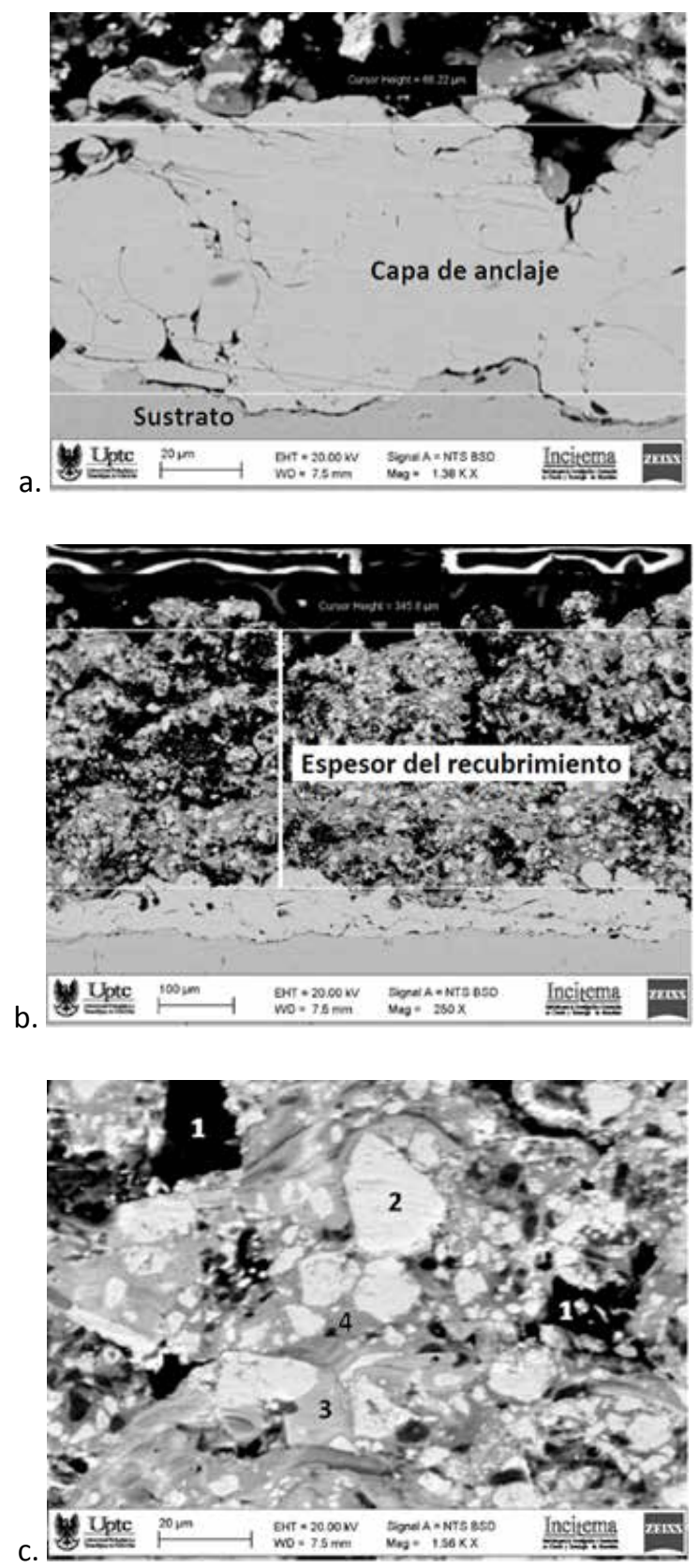

Figura 11. Análisis por SEM de la aplicación de recubrimiento. a) Sustrato y capa de anclaje. b) espesor de la capa de recubrimiento. c) Características propias del recubrimiento

\section{AGRADECIMIENTOS}

El trabajo corresponde con los resultados del proyecto: Optimización de un sistema de recubrimiento cerámico por spray térmico, código SGI 1526 UPTC, financiado por la convocatoria DIN No 3 de 2014 Capital Semilla.

Los autores expresan sus agradecimientos a:

La Dirección de Investigaciones DIN de la Universidad Pedagógica y tecnológica de Colombia, por los aportes al desarrollo del proyecto a partir de recursos de Capital Semilla.

Instituto para la investigación e innovación en ciencia y tecnología de materiales - INCITEMA de la Universidad Pedagógica y Tecnológica de Colombia.

Centro de Gestión de Investigación y Extensión CIFAD. UPTC. Facultad Seccional Duitama.

Los estudiantes Rafael Ramírez Matiz y Helio Dávila por su aporte al desarrollo del proyecto.

\section{REFERENCIAS}

Abello Linde, S.A., (2002). Proyección Térmica de Superficies. pp. 2-15, España. Recuperado de http:// www.abellolinde.es/internet.lg.Ig.esp/es/images/LINSPRAY\%20proyecci\%C3\%B3n\%20t\%C3\%A9rmica\%20de\%20superficies\%20130490207316_60056.PDF

ASM. (2003). Corrosion: Fundamentals, Testing, and Protection. Handbook Volume 13. ASM International. Ohio.

Davis, J., (2004). Introduction to Thermal Spray Processing, Handbook of Thermal Spray Technology. ASM International. Ohio.

García, F., Cuetos J., (2001). Recubrimientos de Proyección por Plasma. Departamento de Construcción e Ingeniería de Fabricación. Universidad de Oviedo. España.

González, A., (2008), Estudio de la influencia de las propiedades físicas en el comportamiento triboló- 
gico de recubrimientos duros para herramientas de corte y procesamiento de polietileno. Tesis de Maestría. Universidad de Antioquia. Medellín.

International Thermal Spray Association, (2015). Thermal Spray. Ohio. Recuperado el 13 de agosto de 2015, de http://www.mecpl.com/pdf-files/ what-is-thermal-spray.pdf.

Marín, C., (2008). Thermal Spray, Protección de alto impacto. Revista Metal Actual. Vol. 8, p. 40-45. Recuperado de: http://www.metalactual.com/ revista/8/tratamiento_thermal_spray.pdf

Marulanda, J., Tristancho, J. y Gonzáles H. (2014). La tecnología de recuperación y protección contra el desgaste está en el rociado térmico. Universidad Tecnológica de Pereira. Pereira. Revista Prospect. Vol. 12, No. 1, p. 70-78. DOI: http:// dx.doi.org/10.15665/rp.v12i1.153

Morales, J., Olaya J. y Rojas H. (2012). Una aproximación a la tecnología de proyección térmica. AVANCES Investigación en Ingeniería Vol. 9 - No. 2, 60-71.
Restrepo, E., Vargas, F., López, M., Cardona, R., Duarte, G. (2012). Elaboración de recubrimientos cerámicos mediante proyección térmica por combustión a partir de residuos sólidos industriales. Universidad de Antioquia. Medellín. Revista Matéria, Vol 17, No. 4, 1176-1185. DOI: http://dx. doi.org/10.1590/S1517-70762012000400008

Ružić, J., Vilotijević, M., Božić, D., Raić K., (2012). Understanding plasma spraying process and characteristics of dc-arc plasma gun (PJ-100). Association of Metallurgical Engineers of Serbia. Vol 18 (4), p. 273-282.

Sulzer, (2013), An Introduction to Thermal Spray. Sulzer Metco. Recuperado el 13 de agosto de 2015, de //www.upc.edu/sct/es/documents_equipament/d_324_id-804-2.pdf

Vargas, F. (2012). Evaluación de la resistencia al choque térmico de recubrimientos cerámicos de $\mathrm{Al} 2 \mathrm{O} 3$ elaborados por proyección térmica. Universidad Industrial de Santander. Bucaramanga. 